\title{
Estimativa da Massa Muscular Esquelética em Mulheres Idosas: Validade da Impedância Bioelétrica
}

\author{
Validity of Bioelectrical Impedance Analysis for the Estimation of \\ Skeletal Muscle Mass in Elderly Women
}

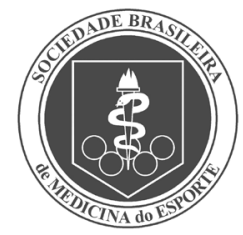

Artigo Original
Cassiano Ricardo Rech

Eline Salomons ${ }^{2}$

Luiz Rodrigo Augustemak de Lima ${ }^{3}$ Edio Luiz Petroski ${ }^{4}$

Maria Fátima Glaner ${ }^{5}$

1. Professor Assistente,

Departamento de Educação Física, Setor de Ciências Biológicas e da Saúde, Universidade Estadual de Ponta Grossa, PR, Brasil, Bolsista

Fundação Arauçaria.

2. Profissional de Educação. Especialização em Treinamento Esportivo, Universidade Federal do Paraná.

3. Mestrando do Programa de Pós-graduação em Educação Física da Universidade Federal de Santa Catarina. Bolsista Capes.

4. Professor Titular, Programa de Pós-graduação em Educação Física da Universidade Federal de Santa Catarina.

5. Professora Adjunta, Programa de Pós-Graduação Stricto Sensu em Educação Física, Universidade Católica de Brasília, Brasília, DF, Brasil.

Endereço para correspondência: Departamento de Educação Física Carlos Cavalcanti, 4.748

Av. Uvaranas - 84030-900 - Ponta Grossa, PR, Brasil

E-mail: crrech@hotmail.com

\section{RESUMO}

O presente estudo tem como objetivos: a) verificar a concordância entre os métodos da impedância bioelétrica (BIA) e da absortometria radiológica de dupla energia (DXA) para a estimativa da massa muscular esquelética (MME); e b) analisar o poder preditivo de variáveis antropométricas e da BIA para a predição da MME em idosas. Foram avaliadas 120 mulheres (60 a 81 anos), residentes na região Sul do Brasil. Mensuraram-se as variáveis antropométricas (massa corporal e estatura); a resistência e hidratação dos tecidos livres de gordura foram medidas pela técnica da BIA tetrapolar (Biodinamics - BF-310), e pela DXA de corpo inteiro (Lunar Prodigy DF + 14319 Radiation e software 7.52.002 DPX-L). A diferença entre os métodos foi verificada pelo teste $t$ pareado, análise dos resíduos e o coeficiente de correlação. O valor preditivo das variáveis antropométricas e de BIA foi verificado pela regressão linear múltipla, adotando nível de significância de $p<0,05$. Não houve diferença estatística entre os métodos para a estimativa da MME $(p>0,01)$. Observou-se que a BIA subestimou em média 0,8kg $\left(\mathrm{C}_{950 \%}:-3,7 ; 2,0 \mathrm{~kg}\right)$ a $M M E$, quando comparada com a DXA. Foi observada alta correlação entre os métodos $\left(r^{2}=0,75 ; p<0,01\right)$. A análise de regressão demonstrou que a variável da razão entre a estatura ao quadrado e a resistência (EST $2 / R$ ) explica $80 \%$ da variação da MME, quando ajustada para massa corporal e idade, e essa relação é independente das variáveis de gordura corporal, hidratação dos tecidos magros e índice de massa corporal. Assim, notase que a equação da BIA, aqui testada, é válida para a estimativa da MME em mulheres idosas e seu valor pode ser mais bem predito pelo modelo de regressão proposto a partir da medida de EST2/R ajustada para a massa corporal e idade.

Palavras-chave: idosos, impedância bioelétrica, densitometria, envelhecimento, composição corporal.

\section{ABSTRACT}

The objectives of the present study were: a) to determine the agreement between bioelectrical impedance analysis (BIA) and dual-energy X-ray absorptiometry (DXA) for the estimation of skeletal muscle mass (SMM), and b) to analyze the predictive power of anthropometric variables and BIA for the prediction of SMM in elderly women. A total of 120 women (60 to 81 years), living in the southern region of Brazil, were studied. Anthropometric variables (body weight and height) were measured. Resistance and hydration of fat-free tissues were measured by tetrapolar BIA (Biodynamics, BF-310) and by whole-body DXA (Lunar Prodigy DF + 14319 Radiation and DPX-L software, version 7.52.002). Differences between methods were determined using the paired t-test, analysis of residuals and correlation coefficient. The predictive value of the anthropometric variables and BIA was evaluated by multiple linear regression, adopting a level of significance of $p<0.05$. No significant difference in the estimation of SMM was observed between methods $(p<0.01)$. BIA underestimated SMM in mean of $0.8 \mathrm{~kg}(95 \% \mathrm{Cl}:-3.7 ; 2.0 \mathrm{~kg})$ when compared to DXA. Correlation between methods was high $\left(r^{2}=0.75 ; p<0.01\right)$. Regression analysis demonstrated that the ratio between the square of height and resistance ( $\mathrm{HEIGHT}^{2} / \mathrm{R}$ ) explained $80 \%$ of the variation in SMM when adjusted to body weight and age, regardless of body fat, lean tissue hydration or body mass index. Thus, the BIA equation tested here is a valid tool for the estimation of SMM in elderly women and its value is best predicted by the regression model including $\mathrm{HEIGHT}^{2} / \mathrm{R}$ adjusted to body weight and age.

Keywords: elderly, bioelectrical impedance, densitometry, aging, body composition. 


\section{INTRODUÇÃO}

A maior expectativa de vida da população e a diminuição da taxa de mortalidade infantil levaram a aumento exponencial da população de idosos, na última década, no Brasil(1). Da mesma forma, o envelhecimento populacional tem sido relatado em países desenvolvidos ${ }^{(2)} \mathrm{e}$ em desenvolvimento(3).

Uma das alterações mais associadas ao envelhecimento é a diminuição da massa muscular esquelética (MME) ${ }^{(4,5)}$, que tem sido relatada como sarcopenia, provocada pela redução da força muscular, aumento do risco de resultados adversos de saúde, como quedas e consequentes fraturas, inaptidão física e perda da autonomia na população idosa ${ }^{(6,7)}$.

Para a estimativa da MME em estudos laboratoriais, têm-se utilizado a ressonância magnética e a tomografia computadorizada como métodos de referência, porém, estes apresentam limitações logísticas e financeiras que inviabilizam sua aplicação em estudos de campo(8).

Recentemente, a absortometria radiológica de dupla energia (DXA) tem ganhado espaço no estudo da estimativa da MME. Investigações indicam que a DXA é um método válido para a estimativa da MME, tanto em homens quanto em mulheres idosas ${ }^{(6,9)}$. Entretanto, mesmo com o desenvolvimento de métodos para estimar a MME, como a DXA, os custos ainda continuam elevados. Nesse sentido, Janssen et al. ${ }^{(10)}$ propuseram uma equação baseada em variáveis de impedância bioelétrica (BIA) e antropometria, para a estimativa da MME em homens e mulheres. A validade dessa equação em homens idosos, na população brasileira, foi evidenciada, recentemente, por Lima et al. ${ }^{(11)}$, demonstrando excelente relação $\left(r^{2}=0,80\right)$ entre as medidas da equação da BIA e a DXA.

A facilidade na coleta de dados por meio da BIA, além de ser um método não invasivo, portátil, rápido e barato para mensurar a resistência corporal, pode incentivar novos estudos sobre a relação entre as alterações da MME (sarcopenia) e envelhecimento(12).

Desse modo, devido à inexistência, no Brasil, de equações de BIA, válidas para estimar a MME em mulheres idosas, o presente estudo tem como objetivo analisar a validade da equação baseada nas variáveis da BIA e antropometria em comparação com a medida da MME por DXA e verificar o poder preditivo das variáveis antropométricas e de BIA para predição da MME em mulheres idosas.

\section{MÉTODO}

Os dados analisados no presente estudo são provenientes do banco de dados originário do estudo de composição corporal em idosos ${ }^{(13)}$. Esta pesquisa seguiu os princípios éticos de respeito à autonomia das pessoas, apontados pela Resolução n 196, de 10 de outubro de 1996, do Conselho Nacional de Saúde. O projeto foi aprovado pelo Comitê de Ética em Pesquisa com Seres Humanos sob nº 059/05.

A coleta de dados foi realizada no ano de 2005, com grupos de atendimento ao idoso da cidade de Florianópolis-SC. Foram considerados como população do estudo todos os idosos inscritos nos Grupos de Terceira Idade de Florianópolis, SC (5.326 idosos). Posteriormente, foram sorteados, aleatoriamente, quatro grupos, totalizando o número de 526 idosos.

Os idosos desses grupos foram listados em ordem alfabética e a seleção da amostra foi realizada por sorteio aleatório sistemático, iniciando pelo primeiro nome da lista; posteriormente, foram selecionados indivíduos com um intervalo a cada nove nomes, proporcionando, assim, a possibilidade de todos participarem da seleção. Foram excluídos do estudo os indivíduos com idade inferior a 60 anos, que apresentassem doenças incapacitantes (paralisias, derrames, câncer, hipertensos, hipertireoidismo, hipotireoidismo, insuficiência renal ou hepática), assim como aqueles que faziam uso contínuo de medicamentos diuréticos, que pudessem interferir na medida da BIA. Os fatores de exclusão foram obtidos por meio de um questionário realizado com o idoso antes da coleta dos dados.

A amostra foi composta por 120 mulheres idosas (60-81 anos), com índice corporal médio de 27,4kg/m² (variando de 18,4kg/m² a 39,3kg/ $\mathrm{m}^{2}$ ), todas residentes no município de Florianópolis, Estado de Santa Catarina, localizado na região Sul do Brasil. O número amostral foi estabelecido de acordo com outros estudos com o mesmo delineamento(10).

Foram mensuradas a massa corporal e a estatura de acordo com a padronização proposta por Gordon et al. ${ }^{(14)}$. Utilizaram-se uma balança Arja, com capacidade para $150 \mathrm{~kg}$ e um estadiômetro de parede com escala de $0,1 \mathrm{~cm}$. A partir dessas medidas, obteve-se o valor do índice de massa corporal.

A medida da MME foi obtida pelo método da DXA por um scanner de corpo inteiro, utilizando um aparelho da marca Lunar Prodigy DF + 14319 Radiation (Madison, WI). A quantificação dos valores de gordura corporal (\%G) e dos tecidos moles do esqueleto apendicular (TMMA) foi realizada com o software DPX-L versão 7.52.002. Os idosos ficaram descalços, sem o uso de anéis, de próteses dentárias e outros tipos de materiais metálicos, usando apenas um avental. Foi solicitado ao idoso deitar-se na mesa em decúbito dorsal, permanecendo imóvel durante a realização da medida de corpo inteiro, que levou de 20 a 30 minutos.

O aparelho foi calibrado diariamente, de acordo com as recomendações do fabricante. As avaliações foram realizadas por uma técnica em radiologia médica, junto ao Centro de Diagnóstico por Imagens (Sonitec), na cidade de Florianópolis-SC, no mesmo dia da coleta das medidas antropométricas. A partir da medida da DXA, foram selecionadas as medidas de tecidos moles do esqueleto apendicular, em quilogramas, além das medidas de idade (anos) e sexo ( 0 = feminino; 1 = masculino), para a estimativa da MME de corpo inteiro, de acordo com o modelo proposto por Kim et al. ${ }^{(9)}$.

$$
\operatorname{MME}(\mathrm{kg})=(1,13 * \mathrm{TMMA})-(0,02 * \text { idade })+(0,61 * \text { sexo })+0,97
$$

Ainda foram coletadas as medidas de impedância bioelétrica (resistência e hidratação do tecido livre de gordura), que foram obtidas por um analisador de composição corporal tetrapolar Biodinamics (modelo BF-310). Esse aparelho emite uma corrente indolor de baixa intensidade de $800 \mu \mathrm{A}$, à frequência de $50 \mathrm{kHz}$, seguindo o caminho do eletrodo fonte até o de captação.

Os eletrodos foram colocados nos pontos anatômicos (mão, punho, tornozelo e pé), todos no hemicorpo direito, estando o indivíduo em decúbito dorsal, em uma superfície não condutora de eletricidade. Foram seguidos os cuidados pré-teste sugeridos por Heyward e Stolarczyk ${ }^{(15)}$. O valor da estatura $(\mathrm{cm})$ ao quadrado e a resistência ( $R$, ohms) foram utilizados para a estimativa da MME de corpo inteiro, por meio do modelo de regressão proposto por Janssen et al. ${ }^{(10)}$, ajustada por sexo (0 = feminino; 1 = masculino) e idade (anos).

$$
\begin{gathered}
\text { MME }(\mathrm{kg})=\left[\left(\text { estatura }^{2} / \text { resistência } \times 0,401\right)+(\text { sexo } \times 3,825)+\right. \\
(\text { idade } x-0,071)]+5,102
\end{gathered}
$$

Para analisar a validade concorrente da técnica da BIA em relação à DXA, para estimar a MME, foram comparadas as médias por meio do teste $t$ pareado, correlação linear de Pearson e coeficiente de determinação $\left(R^{2}\right)$. Ainda foram analisados a diferença média (DM) e o erro padrão de estimativa (EPE) entre as técnicas e análise da dispersão dos resíduos ${ }^{(16)}$.

A análise multivariada foi realizada a fim de verificar o valor preditivo das variáveis de massa corporal ( $\mathrm{kg})$, índice de massa corporal $\left(\mathrm{kg} / \mathrm{m}^{2}\right)$, percentual de gordura corporal (\%G), hidratação dos tecidos livres de gordura (\%), índice da razão entre a estatura ao quadrado e a resistência (EST2/R) para estimar MME. Para a análise dos dados, foi utilizado o programa Statistical Package for the Social Sciences (SPSS) versão $13.0(p<0,05)$.

\section{RESULTADOS}

As idosas apresentaram média de idade de 69,3 anos (DP = 5,7 anos), com amplitude de 21 anos (60-81 anos). A partir dos valores de índice de massa corporal (IMC) foi possível observar que a amostra apresentou prevalência de 7,6\% de indivíduos com baixo peso, 55,9\% 
peso normal e 36,4\% com excesso de peso, de acordo com as recomendações para idosos ${ }^{(17)}$; porém, nota-se que a amostra contou com idosas de todas as categorias de estado nutricional, o que permite validade externa maior dos dados aqui analisados. A mesma variabilidade foi observada nos valores \%G, nos quais as idosas apresentaram variação entre 15,6\% e 51,4\%, valores estes mensurados por meio da DXA. Outros resultados referentes às características antropométricas da amostra estão apresentados na tabela 1.

Tabela 1. Valores descritivos das variáveis antropométricas e de composição corporal de mulheres idosas.

\begin{tabular}{|c|c|c|c|c|}
\hline Variáveis $(n=120)$ & Média & $\mathrm{DP}$ & Mínimo & Máximo \\
\hline Massa corporal (kg) & 65,6 & 11,1 & 39,8 & 107 \\
\hline Estatura $(\mathrm{cm})$ & 154,5 & 5,7 & 141 & 172 \\
\hline IMC $\left(\mathrm{kg} / \mathrm{m}^{2}\right)$ & 27,4 & 3,9 & 18,4 & 39,3 \\
\hline$\% G_{D X A}$ & 37,3 & 6,9 & 15,6 & 51,4 \\
\hline$M L G_{D X A}(k g)$ & 38,8 & 4,5 & 26,2 & 52,8 \\
\hline Resistência ${ }_{\mathrm{BIA}}(' \Omega)$ & 557,3 & 56,8 & 415,1 & 680,5 \\
\hline Hidratação MLG ${ }_{\text {BIA }}(\%)$ & 30,9 & 3,5 & 23,4 & 42,1 \\
\hline
\end{tabular}

IMC: índice de massa corporal; \% $\mathrm{G}_{\mathrm{DXA}}$ : percentual de gordura corporal mensurada pela absortometria radiológica de dupla energia (DXA); MLG DXA: massa livre de gordura mensurada pela DXA; BIA: impedância bioelétrica; DP: desvio padrão.

A tabela 2 apresenta os resultados referentes à validade concorrente da equação de BIA em relação à DXA, para estimar a MME em mulheres idosas. Não houve diferença significativa $(p>0,01)$ entre as médias, sendo a $D M=0,8 \mathrm{~kg}$. A correlação entre os métodos foi de $r$ $=0,85(p<0,001) \cdot O R^{2}$ indica que $75 \%$ da MME BIA foram explicadas pela MME por meio da DEXA.

Tabela 2. Análise da validade concorrente da BIA para a estimativa da massa muscular esquelética em mulheres idosas.

\begin{tabular}{c|c|c|c|c|c}
\hline Métodos & Média & DP & $\mathbf{r}$ & $\mathbf{R}^{2}$ & DM \\
\hline $\mathrm{MME}_{\mathrm{DXA}}$ & 18,5 & 0,24 & \multirow{2}{*}{$0,85^{*}$} & $0,75^{*}$ & $-0,8^{* *}$ \\
\hline $\mathrm{MME}_{\mathrm{BIA}}$ & 17,7 & 0,21 & & & \\
\hline
\end{tabular}

MME: massa muscular esquelética (kg); $\mathrm{DM}=$ diferença média $\left(\mathrm{MME}_{\mathrm{BIA}}-\mathrm{MME}_{\mathrm{DXA}}\right)$; $\mathrm{DP}$ : desvio-padrão; ${ }^{*} \mathrm{p}<0,001$; r: coeficiente de correlaçăo; $R^{2}$ : Coeficiente de determinaçăo; **não houve diferença (teste $t$ pareado),

Houve alta correlação entre os métodos, demonstrando que a equação da BIA representa a MME e explica em 75\% a variação dessa variável quando mensurada pela DXA. Os desvios padrões entre ambos os métodos também foram próximos, indicando que a amplitude dos valores medidos pelo método BIA para a estimativa da MME foi aproximada aos do método DXA.

A figura 1 indica que o erro médio entre DXA e BIA foi de 0,8kg $\left(\mathrm{CC}_{95 \%}:-3,7: 2,0 \mathrm{~kg}\right)$.

Com base nos resultados atribuídos aos resíduos é possível observar que $82,5 \%(n=99)$ das idosas apresentaram um erro de estimativa da MME pelo método da BIA entre -2 e $2 \mathrm{~kg}$. Quando se reduz essa margem de erro $(-1$ e $1 \mathrm{~kg})$, observa-se que $49,2 \%(n=59)$ das idosas estão nesse intervalo de erro (dados não apresentados).

A tabela 3 apresenta a relação entre as variáveis antropométricas (massa corporal, IMC), \%G, hidratação da MLG, EST²/R e a técnica da BIA com o objetivo de verificar o poder preditivo da estimativa da MME. Através da análise univariada, observou-se que o \%G não apresentou correlação significativa com a MME $\left(r^{2}=0,03\right)$. A variável de $E S T^{2} / R$ teve a maior correlação e por isso é a que melhor representa a $\operatorname{MME}\left(r^{2}=0,75\right)$.

No modelo multivariado, foram incluídas as variáveis de $E S T^{2} / R$, hidratação da MLG, massa corporal, e IMC, nessa ordem de inclusão. A variável de \%G não foi incluída no modelo por não apresentar significância estatística ( $p>0,05)$, conforme apresentado na tabela 3.

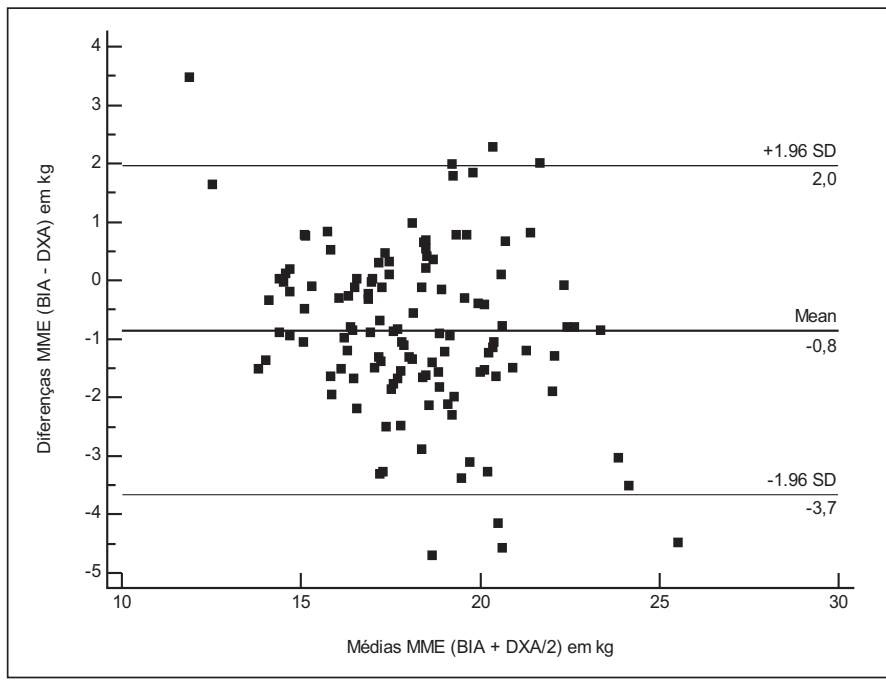

Figura 1. Análise dos escores residuais da massa muscular esquelética (MME) obtida pela DXA e impedância bioelétrica em mulheres idosas. A linha contínua representa o erro médio e a linha contínua representa o erro médio e o intervalo de confiança (95\%).

Tabela 3. Valores preditivos das variáveis antropométricas e da impedância bioelétrica para a estimativa da massa muscular em mulheres idosas.

\begin{tabular}{l|c|c|c}
\hline \multicolumn{1}{c|}{ Variáveis } & $\mathbf{r}$ & $\mathbf{R}^{2}$ & $\mathbf{p}$ \\
\hline Massa corporal $(\mathrm{kg})$ & 0,73 & 0,53 & 0,000 \\
\hline $\mathrm{IMC}\left(\mathrm{kg} / \mathrm{m}^{2}\right)$ & 0,54 & 0,29 & 0,000 \\
\hline \%Gordura & 0,16 & 0,03 & $\mathrm{~ns}$ \\
\hline Hidratação* (\%) & 0,84 & 0,73 & 0,000 \\
\hline EST $^{2} / \mathrm{R}$ & 0,85 & 0,75 & 0,000 \\
\hline
\end{tabular}

*Hidratação dos tecidos livres de gordura; IMC: índice de massa corporal; Estatura²/Resistência: (EST)²/R; ns: não significativo; r: coeficiente de correlação; $R^{2}$ : Coeficiente de determinação.

Na tabela 4, são apresentados os modelos de regressão linear entre as variáveis antropométricas e de BIA para a estimativa da MME. As variáveis de hidratação da MLG e IMC não foram significativas $(p<0,05)$ quando inseridas no modelo multivariado. Esse fato deve-se, em parte, a essas variáveis possuírem covariância com as variáveis $E S T 2 / R(r=0,90)$ e massa corporal $(r=0,88)$. A variável EST2R explicou $75 \%$ da variação da MME (modelo 1), com um erro padrão de estimativa de 1,46kg. Essa correlação foi maior quando ajustada para a massa corporal $\left(r^{2}=0,78\right)$ (modelo 2) e para a massa corporal e idade $\left(r^{2}=0,80\right)$ (modelo 3).

Tabela 4. Modelos de regressão linear para a estimativa da massa muscular esquelética em mulheres idosas, a partir de variáveis antropométricas e de impedância bioelétrica.

\begin{tabular}{l|c|c|c|c}
\hline Modelos de regressão & $\beta$ & $\mathbf{r}$ & $\mathbf{R}^{2}$ & EPE (kg) \\
\hline Modelo 1 & \multicolumn{5}{|l}{} \\
\hline (EST) $)^{2} / \mathrm{R}$ & 0,403 & & & \\
\hline Constante & 1,084 & 0,85 & 0,75 & 1,46 \\
\hline Modelo 2
\end{tabular}

\begin{tabular}{l|c|c|c|c}
\hline Modelo 2 \\
\hline$(\text { EST) })^{2} / \mathrm{R}$ & 0,308 & & & \\
\hline Massa corporal (kg) & 0,07307 & & & \\
\hline Constante & 0,425 & 0,88 & 0,78 & 1,34 \\
\hline
\end{tabular}

\section{Modelo $3^{*}$}

\begin{tabular}{l|c|l|l|l}
\hline$(\text { EST) })^{2} / \mathrm{R}$ & 0,311 & & & \\
\hline Massa corporal (kg) & 0,06994 & & & \\
\hline Idade (anos) & $-0,0452$ & & & \\
\hline Constante & 3,526 & 0,90 & 0,80 & 1,31 \\
\hline
\end{tabular}

* Independente da hidratação da massa livre de gordura e do índice de massa corporal; EPE: erro padrão de estimativa; EST2/R: estatura²/resistência. 
A equação que segue (modelo 3) estima a MME em homens idosos, apresentando a estatura (EST) em centímetros, resistência (R) em ohms; a massa corporal (MC) é expressa em quilogramas e a idade (ID) em anos.

$\operatorname{MME}(\mathrm{kg})=3,526+\left[\left(\mathrm{EST}^{2} / \mathrm{R}^{*} 0,311\right)+(0,06994 * \mathrm{MC})-(0,0452 * \mathrm{ID})\right]$

Assim, os resultados demonstram que a MME pode ser estimada pelas variáveis de BIA e antropometria quando ajustadas pela massa corporal (kg) e idade (anos), explicando 80\% da variação da MME e com um EPE de 1,3kg.

\section{DISCUSSÃO}

O processo de envelhecimento tem como consequência perda de massa muscular esquelética em decorrência da diminuição da atividade física, facilitando, assim, o aparecimento de problemas fisiológicos, de doenças crônicas e problemas metabólicos (obesidade, diabetes, doenças cardiovasculares, etc.) $)^{(5,18,19)}$.

Alguns estudos têm como objetivo analisar a ocorrência da sarcopenia na população de idosos ${ }^{(4,7,20)}$. Porém, para que mais estudos populacionais sejam realizados, são necessários métodos que apresentem validade, baixos custos e facilidades operacionais para coleta de dados.

A BIA é atualmente o método mais utilizado para determinação da massa muscular de idosos pela sua alta velocidade no processamento das informações, por ser um método não invasivo e relativamente barato ${ }^{(21)}$ e também válido em diferentes grupos populacionais ${ }^{(6,10,11,22)}$. Portanto, a BIA vem a ser um método válido para essa estimativa.

No presente estudo, os resultados demonstram que não houve diferença $(p>0,01)$ entre as médias da MME nos métodos propostos, DXA e BIA, para mulheres idosas. A técnica da BIA baseia-se, especificamente, na condução de baixa corrente elétrica pelos tecidos magros. Assim, quanto maior a quantidade de MME, maior será a condutibilidade e menores os valores da resistência à corrente elétrica ${ }^{(23)}$.

Isso pode explicar por que o índice EST2/R tem se mostrado como uma excelente variável para predizer a MME $\left(R^{2}=75 \%\right)$, quando utilizada a técnica da BIA ${ }^{(10)}$. No presente trabalho, a variável EST2/R explicou $75 \%$ da variação da MME estimada pela BIA. Esses resultados estão de acordo com os dados encontrados por Janssen et al.(4), observando que as variáveis da BIA combinadas com medidas antropométricas podem predizer a MME.

\section{REFERÊNCIAS}

1. Carvalho JAM, Rodríguez-Wong LL. A transiçăo da estrutura etária da populaçăo brasileira na primeira metade do século XXI. Cad Saúde Pública 2008;24:597-605.

2. World Health Organization. Active ageing: a policy framework. Geneva, Switzerland, 2002.

3. Organizaçăo Pan-americana de Saúde (OPAS). Health situation in the Americas: basic indicators. Washington, DC; 2006.

4. Janssen I, Baumgartner RN, Ross R, Rosenberg IH, Roubenoff R. Skeletal muscle cutpoints associated with elevated physical disability risk in older men and women. Am J Epidemiol 2004;159:413-21.

5. Dey DK, Bosaeus I. Comparison of bioelectrical impedance prediction equations for fat-free mass in a population-based sample of 75 y olds: The NORA Study. Nutrition 2003;19:858-64.

6. Chen Z, Wang Z, Lohman T, Heymsfield SB, Outwater E, Nicholas JS, et al. Dual-energy x-ray absorptiometry is a valid tool for assessing skeletal muscle mass in older women. J Nutr 2007;137:2775-80.

7. Baumgartner RN, Koehler KM, Gallagher D, Romero L, Heymsfield SB, Ross RR, et al. Epidemiology of sarcopenia among the elderly in New Mexico. Am J Epidem 1998;147:755-63.

8. Heyward, V. ASEP methods recommendation: body composition assessment. J Exerc Physiol Online 2001;4:1-12.

9. Kim J, Wang Z, Heymsfield SB, Baumgartner RN, Gallagher D. Total body skeletal muscle mass: estimation by a new dual-energy x-ray absorptiometry method. Am J Clin Nutr 2002;76;378-83.

10. Janssen I, Heymsfield SB, Baumgartner RN, Ross R. Estimation of skeletal muscle mass by bioelectrical impedance analysis. J Appl Physiol 2000;89:465-71.

11. De Lima LRA, Rech CR, Petroski EL. Utilização da impedância bioelétrica para estimativa da massa muscular esquelética em homens idosos. Arch Latinoam Nutr 2008:58:123-31.

12. Norgan NG. Laboratory and field measurements of body composition. Public Health Nutr 2005;8:1108-22.
É importante salientar que os erros na estimativa de MME pelos métodos DXA e BIA (figura 1) sugerem que as variações da MME não são extremas, pois $95 \%$ da amostra apresentam um erro entre -3,7kg e 2,0kg (variação de 5,7kg), notando-se, assim, que a equação proposta por Janssen et al.(10) pode ser utilizada para estimar a MME em idosas.

Em relação ao valor preditivo das variáveis antropométricas e de BIA para estimar a MME, observou-se que a variável de EST2/R foi a que melhor representou a MME $\left(R^{2}=0,75 ; E P E=1,46 \mathrm{~kg}\right)$. Quando inseridas as variáveis de massa corporal e idade, esse modelo foi ajustado e proporcionou uma explicação de $80 \%$ da MME mensurada pela DXA e reduzindo o EPE para 1,3kg. Isso demonstra que as variáveis de massa corporal e idade são importantes ajustes a serem considerados na avaliação da MME.

A relação da MME com a idade e massa corporal é evidenciada nos estudos apresentados por Nair (24), pois com o envelhecimento existe diminuição da MME e variações na massa corporal que necessitam ser ajustadas no modelo.

Por outro lado, as variáveis de gordura corporal, hidratação dos tecidos livres de gordura e IMC, não apresentaram significância estatística ( $p>0,05)$, para estimar a MME. O \%G não apresentou relação com a MME na análise univariada e não foi incluído no modelo de ajuste.

As variáveis de hidratação dos tecidos livres de gordura e IMC não apresentaram significância estatística no modelo multivariado por possuir covariância com as variáveis de $E S T^{2} / R(r=0,90)$ e massa corporal $(r=0,88)$, que não foram significativas no modelo de ajuste.

Portanto, o método BIA pode ser mais bem explorado para a estimativa da MME com equações que levem em consideração as variações da massa corporal e idade, independente da quantidade de gordura corporal, hidratação dos tecidos livres de gordura e IMC.

Assim, pode-se sugerir, a partir dos dados apresentados no presente estudo, que existe forte correlação entre os métodos da DXA e da $B I A^{(10)}$ para estimar a MME em mulheres idosas. Sugere-se a validade cruzada do modelo de BIA proposto por Janssen et al.(10). A EST²/R é um ótimo preditor da MME, quando ajustada para massa corporal e idade, independente da quantidade de gordura corporal, hidratação dos tecidos livres de gordura e IMC.

O presente estudo foi aprovado pelo Comitê de Ética (processo $\left.n^{\circ} 25 / 2005\right)$.

Todos os autores declararam não haver qualquer potencial conflito de interesses referente a este artigo.
13. Rech CR. Validação de equações antropométricas e de impedância bioelétrica para a estimativa da composição corporal em idosos [Dissertação de Mestrado]. Universidade Federal de Santa Catarina; 2006.

14. Gordon CC, Chumlea WC, Roche AF. Stature, recumbent length, and weight. In: Lohman TG, et al., editors. Anthropometric standardization reference manual. Champaign: Human Kinetics Books; 1991. p. 3-8.

15. Heyward VH, Stolarczyk LM. Avaliação da composiçăo corporal aplicada. São Paulo: Manole; 2000.

16. Bland JM, Altman DG. Statistical methods for assessing agreement between two methods of clinical measurement. Lancet 1986;1:307-10.

17. Lipschitz DA. Screening for nutrition status in the elderly. Prim Care 1994;21:55-67.

18. Chumlea WC, Guo SS, Kuczmarski RJ, Flegal KM, Johnson CL, Heymsfield SB, et al. Body composition estimates from NHANES III bioelectrical impedance data. Int J Obes 2002;26:1596-609.

19. Poortmans JR, Boisseau N, Moraine J-J, Moreno-Reyes R, Goldman S. Estimation of total-body skeletal muscle mass in children and adolescents. Med Sci Sports Exerc 2005;37:316-22.

20. Hughes VA, Frontera WR, Roubenoff R, Evans WJ, Singh MA. Longitudinal changes in body composition in older men and women: role of body weight change and physical activity. Am J Clin Nutr 2002;76:473-81.

21. Kyle UG, Bosaeus I, De Lorenzo AD, Deurenberg P, Elia M, Manuel Gómez J, et al. Bioelectrical impedance analysis-part II: utilization in clinical practice. Clin Nutr 2004;23:1430-53.

22. Kushner RF, Schoeller DA, Fjeld CR, Danford L. Is the impedance índex (ht2/R) significant in predicting total body water? Am J Clin Nutr 1993;56:835-9.

23. Wagner DR, Heyward VH. Techniques of body composition assessment: a review of laboratory and field methods. Res Q Exerc Sport 1999;70:135-49.

24. Nair KS. Aging muscle. Am J Clin Nutr 2005;81:953-63. 